Aim of the study was to assess the results of surgical treatment of cervical recurrences in patients with thyroid cancer.

Material and methods: We assessed 66 reoperations of thyroid cancer recurrences in 51 patients. Reoperative surgeries covered I-VII neck levels.

Results and conclusions: The localization of cervical recurrence and the number of removed nodes did not depend on the type of thyroid cancer. Metastatic spread was predominantly observed in the central neck. Bilateral changes tended to be observed more often in young er patients $(p=0.07)$. Radical neck dissections were performed more often at younger age $(p<0.01)$. Postoperative vocal cord paresis was noted in 13 patients; in 5 permanent tracheotomy was necessary, and 2 underwent laser glottis procedures (posterior cordectomies). Two patients died in the postoperative period -1 due to chylothorax and 1 due to severe bleeding from the common carotid artery.

Key words: thyroid cancer, neck dissection.

Contemp Oncol (Pozn) 2014; 18 (4): 268-272 DOI: $10.5114 /$ wo.2014.44627

\section{Evaluation of outcomes after reoperative neck dissection due to thyroid cancer}

\author{
Małgorzata Wierzbicka ${ }^{1}$, Elżbieta Waśniewska-Okupniak ${ }^{1}$, \\ Jacek Banaszewski ${ }^{1}$, Maciej Pabiszczak ${ }^{1}$, Tomasz Piorunek ${ }^{2}$
}

${ }^{1}$ Department of Otorhinolaryngology, Head and Neck Surgery, Poznan University of Medical Sciences, Poznan, Poland

2Department of Pulmonology, Alergology and Respiratory Oncology, Poznan University of Medical Sciences, Poznan, Poland

\section{Introduction}

Increased mortality in patients with thyroid cancer (TC) due to regional lymph node metastases has been previously well described [1, 2]. The necessity of prophylactic neck dissection and the extent of therapeutic neck dissection in this population still remain an unresolved issue [3, 4]. Metastases of thyroid cancer in cervical lymph nodes usually can be easily localized with an imaging test, most often with neck ultrasound [5]. Numerous authors have analyzed various methods to avoid nodal recurrences and proved the efficacy of central compartment dissection [6-8], prophylactic lymphadenectomy of neck level II [9] or even routine dissection of level $V$ in patients with lateral neck metastases [3]. However, few papers have evaluated the feasibility and efficacy of reoperations in this regions $[6,10]$.

Reoperative surgery of the central and lateral neck in TC is associated with a high risk of complications and lower probability of tumor eradication. The question arises, how to deal with patients with different TC histology, who have already been operated on or even reoperated for relapse, or received other treatment, such as radioiodine therapy or radiotherapy, but with no sufficient response. Moreover, it is important to assess the surgical feasibility and morbidity in patients with recurrences in the lower neck anatomical region. The aim of the study was to assess the outcomes of surgical treatment in gross cervical recurrences of thyroid cancer as well as the rate of complications and to describe the surgical traps.

\section{Material and methods}

The study was conducted in a tertiary university centre (Department of Otorhinolaryngology, Head and Neck Surgery, Poznan University of Medical Sciences). Out of 733 neck dissections performed between January 2003 and December 2011, 66 were reoperations due to thyroid cancer recurrences. Cervical masses were diagnosed in 51 consecutive patients with recurrent thyroid cancer (11 patients reoperated more than once). There were $32 \mathrm{fe}$ male and 19 male patients, aged $48 \pm 17$ years and $49 \pm 17$ respectively. The patients' characteristics are presented in Table 1.

The patients enrolled in the study had undergone previous operations for TC in 11 different general surgery units and then followed up in the Department of Endocrinology, Metabolism and Internal Medicine of Poznan University of Medical Sciences. All treatment failures were subsequently sent to the referral head and neck cancer center - the Department of Otorhinolaryngology, Head and Neck Surgery, Poznan Medical University. Recurrent or persistent neck disease was defined as: positive imaging on ultrasound, computed tomography or radioiodine whole body scan, increased thyroglob- 
Table 1. Clinical characteristics of the study group

\begin{tabular}{|c|c|c|c|c|c|c|c|c|}
\hline \multirow[t]{2}{*}{ Histology } & \multirow{2}{*}{$\begin{array}{c}\text { No. of } \\
\text { patients }\end{array}$} & \multicolumn{2}{|c|}{ Gender } & \multirow[t]{2}{*}{ Mean age } & \multicolumn{4}{|c|}{ Primary tumor stage } \\
\hline & & $M$ & $\mathrm{~F}$ & & $\mathrm{~T} 1-\mathrm{T} 2$ & T3-T4 & $\mathrm{N}-$ & $\mathrm{N}+$ \\
\hline papillary & 28 & 6 & 22 & 46 & 11 & 17 & 20 & 7 \\
\hline oxyphilic & 3 & 2 & 1 & 61 & 0 & 3 & 3 & 0 \\
\hline follicular & 2 & 1 & 1 & 34 & 0 & 2 & 1 & 1 \\
\hline medullary & 15 & 8 & 7 & 49 & 11 & 4 & 9 & 6 \\
\hline poorly differentiated & 3 & 1 & 2 & 57 & 0 & 3 & 3 & 1 \\
\hline total & 51 & 19 & 32 & 48 & 22 & 29 & 36 & 15 \\
\hline
\end{tabular}

Table 2. The neck characteristics of patients with thyroid cancer node recurrence and surgical technique

\begin{tabular}{|c|c|c|c|c|c|c|c|c|c|c|c|c|c|c|c|c|}
\hline \multirow[t]{2}{*}{ Histology } & \multirow{2}{*}{$\begin{array}{c}\text { No. of } \\
\text { patients }\end{array}$} & \multicolumn{7}{|c|}{ Neck level } & \multicolumn{2}{|c|}{ Stage } & \multicolumn{4}{|c|}{ Type of surgery } & \multirow{2}{*}{\multicolumn{2}{|c|}{$\begin{array}{c}\text { No. of } \\
\text { procedures }\end{array}$}} \\
\hline & & VI & I & II & III & IV & V & VII & rN1a & rN1b & SND & RND & CC & VII & & \\
\hline papillary & 28 & 8 & 1 & 20 & 22 & 23 & 14 & 7 & 0 & 28 & 19 & 15 & 8 & 7 & 24 & 4 \\
\hline oxyphilic & 3 & 1 & 0 & 1 & 2 & 2 & 0 & 0 & 0 & 3 & 3 & 0 & 1 & 0 & 3 & 0 \\
\hline follicular & 2 & 0 & 0 & 2 & 2 & 1 & 1 & 0 & 0 & 2 & 2 & 0 & 0 & 0 & 2 & 0 \\
\hline medullary & 15 & 4 & 1 & 11 & 12 & 13 & 4 & 1 & 0 & 15 & 11 & 6 & 4 & 1 & 9 & 6 \\
\hline $\begin{array}{l}\text { poorly } \\
\text { differentiated }\end{array}$ & 3 & 1 & 1 & 2 & 2 & 2 & 2 & 2 & 1 & 2 & 2 & 1 & 1 & 2 & 2 & 1 \\
\hline total & 51 & 14 & 3 & 36 & 40 & 41 & 21 & 10 & 1 & 50 & 37 & 22 & 14 & 10 & 40 & 11 \\
\hline
\end{tabular}

SND - selective neck dissection, RND - radical neck dissection, CC - central compartment dissection, VII - mediastinal tumor excision

ulin or calcitonin plasma levels. In the majority of cases the recurrence had been previously confirmed by the results of fine needle aspiration biopsy.

The patients' medical histories were analyzed and the postoperative charts from the first treatment were reviewed. The age of the patients at the onset of disease ranged from 16 to 76 years (mean 48 years). Forty-six patients previously had had one surgical procedure for thyroid cancer and 5 patients two or more. The histology of the primary lesion was determined according to the World Health Organization histological classification and was as follows: 28 cases of papillary cancer (54.9\%), 3 cases of oxyphilic variant of papillary cancer (5.9\%), 2 cases of follicular cancer (3.9\%), 15 cases of medullary cancer (29.4\%) and 3 cases of poorly differentiated thyroid cancer (5.9\%). Staging was performed according to the American Joint Committee on Cancer Classification (AJCC) Cancer Staging Manual revised in 2009 [11]. The stage of the primary tumor was T1 in 6 cases, T2 in 16 cases, T3 in 18 cases and $\mathrm{T} 4$ in 11 cases. Nodal disease at first presentation was NO in 36 (60.8\%), and $\mathrm{N}+$ in 15 (39.2\%) patients. The median time between the primary surgery and reoperation was 2 years (range: $7-16$ years).

All reoperations were carried out with therapeutic intent. The reoperative surgery covered neck levels I-VII and the choice of the procedure depended on $\mathrm{rN}$ advancement detected in imaging, location of macrometastases and tumor histology. The patients' data concerning neck relapse are presented in Table 2.
Radical neck dissection (RND) included the lateral node compartment: jugular chain nodes (levels I, II, III, IV) and posterior neck (level V), from the skull base to the subclavian vein and laterally to the trapezius muscle. Selective neck dissection (SND) was performed at certain nodal levels suspected of malignancy. Selective neck dissection included levels IIa, III, IV, V. In patients who underwent more extensive clearance at the initial surgery, the chosen levels were operated. Dissection of the central compartment (CC) included level VI nodes extending vertically from the hyoid bone to the thoracic inlet and horizontally between the carotid sheaths (prelaryngeal, pretracheal and paratracheal nodes).

Indirect laryngoscopy and stroboscopy were performed before and after the reoperation (on the $1^{\text {st }}$ and $7^{\text {th }}$ day and 6 months later if needed). Seven patients had had unilateral vocal cord paralysis prior to neck dissection.

Serum calcium level and intact parathormone concentration (iPTH) were assessed to identify patients with hypocalcemia.

Student's t test was used for statistical analysis of continuous variables. Categorical variables were analyzed with Fisher's exact test and the $\chi^{2}$ test. $P$-value $<0.05$ was used to define statistical significance.

The weak point of the study was the disperse and lacking documentation of the first line treatment. The strong one was the prospective evaluation of recurrent thyroid cancer cervical mass and all the consequences of introduced treatment. The mean follow-up time after the neck reoperation was $3.4 \pm 2.3$ years. 
The project was carried out with the approval of the Local Ethics Committee.

\section{Results}

Radical neck dissection was performed in 22 patients (43.1\%) and SND in 29 (56.9\%). The central node compartment (level VI) was cleaned in 14 patients (27.5\%) - in 2 of them as the main procedure and as complementary to lateral node compartment dissection in 12 cases. Additionally to RND, SND or CC dissection upper mediastinal nodes (level VII) were dissected in 10 patients (19.6\%). Eleven patients were reoperated more than once (Table 2). Histopathological examination was performed in all cases.

The advancement of nodal recurrence ( $\mathrm{rN})$, tumor histology, precise localization of metastases and type of surgery are presented in Table 2. Stage rN1a and rN1b were noted in 1 and 50 patients respectively.

In 2 patients we observed very aggressive course of the relapse with gross infiltration of the larynx and esophagus, where only humiliating total laryngectomy with partial esophagectomy could have been taken into consideration. Additionally, 1 patient with papillary cancer recurrence required tracheal resection. In these 3 cases the localization of relapse (metastatic node or thyroid cancer remnant) was burdened by high loco-regional advancement.

The analysis of all performed neck dissections revealed that the localization of recurrence (level I-III, IV, V, VI, VII, thyroid remnants) and the time between the primary surgery and the recurrence did not depend on patients' gender, age or the type of thyroid cancer.

The number of removed lymph nodes (2 categories: 1-4 and more than 4) did not depend on patients' gender and the type of thyroid cancer. However, patients requiring dissection of more than 4 nodes were older $(p<0.01)$.

Metastatic spread was predominantly observed in the central neck. Central metastatic nodes were confirmed in 14 cases (27.4\%) and mediastinal metastases at level VII were found in 10 cases (19.6\%). In both localizations this number constituted $100 \%$ of removed nodes; there were no false negative findings. Out of 59 lateral neck specimens metastases were found at levels II and III in 37 cases (72\%) and at level IV in 35 cases (68\%).

The number of metastatic nodes removed during neck dissection ranged from 1 to 28: single metastatic lymph nodes in 11 patients (21.6\%), 2 in 10 patients (19.6\%), 2 in one patient (2\%), 4 in 3 patients (5.9\%) and 5 or more in 25 patients $(47.1 \%)$.

Bilateral neck metastases were found in 17 patients (33.3\%). The presence of bilateral metastases did not depend on patients' gender or the type of thyroid cancer. However, they tended to be observed more often in young patients (below 45 years) $(p=0.07)$ and in patients with primary surgery performed at young age $(p=0.049)$. Furthermore, these two groups more frequently required radical neck dissection (young patients $-p<0.01$, patients with primary surgery performed at young age $-p<0.01$ ). This could be partially explained by the concern in older patients to preserve safety rather than to perform radical surgery at all costs.
The technical difficulties were as follows: in all cases scars, in approximately 30\% disturbed anatomy, in 5 cases infiltration of subclavicular vessels or of the venous angle area, in 2 cases infiltration of the larynx or esophagus, and in 10 cases metastatic mass in the mediastinum. Postoperative complications were divided into major, requiring surgical intervention (bleeding, chylorrhea, dyspnea), and minor (hypocalcemia, unilateral or bilateral vocal cord paresis).

Severe dyspnea occurred in 6 patients: in 3 cases laterofixation and in 3 cases emergency tracheotomy was necessary. In all patients indirect laryngoscopy and stroboscopy were performed the day after surgery. Postoperative vocal cord paresis was observed in 13 patients (25.5\%); in 5 of them it was transient. Direct tumor invasion to the recurrent laryngeal nerve was observed in 7 cases - in 3 patients with medullary cancer, who underwent intentional sectioning and in 4 patients (WDTC) with nerve detached from the tumor. In 6 other patients this structure was not visible in stiff scars, although nerve paresis occurred postoperatively. Five patients required permanent tracheostomy - in 3 cases due to vocal cord paresis and in 2 cases due to total laryngectomy. In a one-year period 2 patients with permanent postoperative bilateral vocal cord paresis required glottis procedures (laser posterior cordectomy according to Kashima) to restore breathing function.

In 7 patients (13.7\%) other intra- or postoperative complications occurred. Four patients had postoperative chyle leakage. In 2 cases it was controlled 6-8 days after surgery. In 1 patient surgical intervention was necessary and in 1 patient chylothorax caused death on the $36^{\text {th }}$ day after surgery. Massive bleeding occurred in 3 patients - in 1 case from the common carotid artery and in 2 cases from the stump of the internal jugular vein.

Hypoparathyroidism was diagnosed in 22 patients (43.1\%), requiring vitamin $\mathrm{D}$ and/or calcium supplementation.

Forty-three procedures (84\%) were assessed subjectively as radical; in 8 patients (15.7\%) the residual tumor was left ( 6 papillary, 1 medullary and 1 anaplastic cancer). These reoperations were performed in the left subclavian or in the upper mediastinal area and were responsible for gross complications - in 5 cases for laryngeal nerve paresis, in 2 patients for chylorrhea, and in 1 patient for chylothorax.

The presence of minor or major postoperative complications did not depend on patients' age, gender, the time of primary surgery, or the type of thyroid cancer. The number of dissected lymph nodes and the presence of bilateral metastatic changes also did not influence the frequency of complications. However, the rate of complications was strictly associated with the number of procedures and with anatomical area (the border of the lower neck and mediastinum (VI/VII) and left vs. right side of level IV.

Distant metastases (to lungs and/or bones) were found in 15 patients. Their occurrence was definitely more frequent in patients with medullary and poorly differentiated thyroid cancer $(p<0.01)$.

\section{Discussion}

In this study we evaluated thyroid cancer relapses (both nodal and local), the interdependence with primary tumor 
histology and primary treatment, the metastasizing pattern, neck surgery salvage technique, the efficacy of reoperations and postoperative complications.

The staging of primary papillary thyroid cancer in this study was determined according to the American Joint Committee on Cancer Classification [11]. Following Mulla and Schulte [8] we also observed that this classification does not emphasize the metastasis process. The American Joint Committee on Cancer (AJCC) classification understaged nearly half of the patients (females $>45$ years old), because stage progression from I or II or III is defined as the presence of lymph node metastasis, except for women $<45$ years old, who remain in stage 1 .

Although the role of prophylactic central lymph node dissection (CLND) in papillary thyroid cancer in subclinical nodal disease is controversial, a higher recurrence rate (16.7\% without CLND vs. $4.4 \%$ ) was proven by Moo et al. [12]. This procedure performed as an addition to total thyroidectomy identified positive nodes in more than 30\% of patients [12-14]. Moreover, recently it has been stated that prophylactic CLND may be performed in papillary cancer with uninvolved central nodes, particularly in advanced primary tumors staged T3-T4 and in the elderly [15-19]. Clearance of the central compartment should be advocated then waiting for nodal recurrence to emerge [7, 13, 14, 19]. Skip metastases (negative central and positive lateral or mediastinal compartments) were found in $19.7 \%$ of cases of papillary and $21.3 \%$ of cases of medullary cancer. Since they are an epiphenomenon of low intensity nodal metastases and entail a moderate risk of local recurrence, clearing the central compartment should be performed, when lateral or mediastinal metastases are present [7].

Sites frequently involved in recurrent or persistent disease include retrocarotid nodes, nodes deep in the laryngeal recurrent nerve, retroclavicular nodes, retrosternal nodes and nodes running along the superior thyroid vascular pedicle medial to the carotid bifurcation [20]. Assessing the pattern of cervical nodal recurrences, we found that the most common sites were levels II and III (43 cases $-84.3 \%$ ) and level IV (41 cases - 80.4\%). Roh et al. [6] found nodal metastases in the central and bilateral central compartment in $86.7 \%$ and $42.2 \%$ of cases, respectively.

In view of the usually mild course of papillary thyroid carcinoma, minimal surgical procedures are considered preferable to radical surgery [14]. A selective approach to lateral cervical node dissection sparing levels I and V unless there is radiological evidence of disease, and a less aggressive approach to level II was suggested [21], but leaving positive nodes in the tracheoesophageal groove may expose the patient to a risk of tracheal, esophageal or even laryngeal invasion [22]. The selection of low- and high-risk patients probably will be in future based on molecular pathology, but before that many patients may be overtreated or, conversely, underestimated [13].

Most centers are univocal that central lymph node clearance in patients with probability of lymph node metastases may avoid reoperative surgery, which is associated with greater morbidity than first-time surgery [8, 9]. However, Sywak et al. [23] demonstrated that there is no additional morbidity, when central lymph node dissection is performed as a secondary procedure. Hartl and Travagli [20] underlined that, although the boundaries and the anatomical structures that require dissection are the same, the scar tissue and neovascularization complicates the access to this region (sternohyoid and sternothyroid muscles are adherent to the trachea in dense scar tissue).

Locating the recurrent laryngeal nerve may be particularly difficult in the case of widespread fibrosis [20]. Thus, reoperative surgery of the central neck is associated with higher risk of vocal fold paresis and permanent hypoparathyroidism (up to 9\%) [6]. In our study long-term follow-up revealed permanent hypoparathyroidism in 22 patients (43.1\%) and recurrent laryngeal nerve injury in 13 cases (25.5\%). Central compartment reoperations according to Mirghani et al. [24] can be performed with minimal morbidity, when the recurrent laryngeal nerve is not invaded (permanent paresis in $5.2 \%$, permanent hypoparathyroidism in $9 \%$ ).

The cut-off point in reoperative neck dissections associated with a higher complication rate is not possible to state clearly. The localization of metastases is crucial for the complication rate.

In conclusion, considering the surgical approach and possible complications, patients with nodal metastatic changes deriving from thyroid cancer present a challenging group for surgeons.

\section{The authors declare no conflict of interest.}

\section{References}

1. Lundgren $\mathrm{Cl}$, Hall P, Dickman PW, Zedenius J. Clinically significant prognostic factors for differentiated thyroid carcinoma: a population-based, nested case-control study. Cancer 2006; 106: 524-31.

2. Tisell LE, Nilsson B, Mölne J, Hansson G, Fjälling M, Jansson S, Wingren $U$. Improved survival of patients with papillary thyroid cancer after surgical microdissection. World J Surg 1996; 20: 854-9.

3. Kupferman ME, Weinstock YE, Santillan AA, Mishra A, Roberts D, Clayman GL, Weber RS. Predictors of level V metastasis in well-differentiated thyroid cancer. Head Neck 2008; 30: 1469-74.

4. Randolph GW. Papillary cancer nodal surgery and the advisability of prophylactic central neck dissection: primum, non nocere. Surgery 2010; 148: 1108-12.

5. Ruchała M, Szczepanek E. Thyroid ultrasound - a piece of cake? Endokrynol Pol 2011; 62 Suppl 1: 8-24.

6. Roh JL, Kim JM, Park Cl. Central compartment reoperation for recurrent/persistent differentiated thyroid cancer: patterns of recurrence, morbidity, and prediction of postoperative hypocalcemia. Ann Surg Oncol 2011; 18: 1312-8.

7. Machens A, Holzhausen HJ, Dralle H. Skip metastases in thyroid cancer leaping the central lymph node compartment. Arch Surg 2004; 139: 43-5.

8. Mulla M, Schulte KM. Central cervical lymph node metastases in papillary thyroid cancer: a systematic review of imaging-guided and prophylactic removal of the central compartment. Clin Endocrinol (Oxf) 2012; 76: 131-6.

9. Koo BS, Seo ST, Lee GH, Kim JM, Choi EC, Lim YC. Prophylactic lymphadenectomy of neck level II in clinically node-positive papillary thyroid carcinoma. Ann Surg Oncol 2010; 17: 1637-41.

10. Shah PK, Shah KK, Karakousis GC, Reinke CE, Kelz RR, Fraker DL Regional recurrence after lymphadenectomy for clinically evident lymph node metastases from papillary thyroid cancer: a cohort study. Ann Surg Oncol 2012; 19: 1453-9.

11. Edge SB, Byrd DR, Compton CC, et al. AJCC Cancer Staging Manual. Springer, New York 2009. 
12. Moo TA, McGill J, Allendorf J, Lee J, Fahey T 3rd, Zarnegar R. Impact of prophylactic central neck lymph node dissection on early recurrence in papillary thyroid carcinoma. World I Surg 2012; 34: 1187-91.

13. Davidson HC, Park BJ, Johnson JT. Papillary thyroid cancer: controversies in the management of neck metastasis. Laryngoscope 2008; 118: 2161-5.

14. Hyun SM, Song HY, Kim SY, Nam SY, Roh JL, Han MW, Choi SH. Impact of combined prophylactic unilateral central neck dissection and hemithyroidectomy in patients with papillary thyroid microcarcinoma. Ann Surg Oncol 2012; 19: 591-6.

15. Costa S, Giugliano G, Santoro L, et al. Role of prophylactic central neck dissection in cNO papillary thyroid cancer. Acta Otorhinolaryngol Ital 2009; 29: 61-9.

16. Cooper DS, Doherty GM, Haugen BR, et al. Revised American Thyroid Association management guidelines for patients with thyroid nodules and differentiated thyroid cancer. Thyroid 2009; 19: 1167214

17. American Thyroid Association Surgery Working Group; American Association of Endocrine Surgeons,; American Academy of Otolaryngology-Head and Neck Surgery; American Head and Neck Society, Consensus statement on the terminology and classification of central neck dissection for thyroid cancer. Thyroid 2009; 19: 1153-8.

18. Moo TA, Umunna B, Kato M, Butriago D, Kundel A, Lee JA, Zarnegar R, Fahey TJ 3rd. Ipsilateral versus bilateral central neck lymph node dissection in papillary thyroid carcinoma. Ann Surg 2009; 250: 403-8.

19. Shindo M, Wu JC, Park EE, Tanzella F. The importance of central compartment elective lymph node excision in the staging and treatment of papillary thyroid cancer. Arch Otolaryngol Head Neck Surg 2006; 132: 650-4.

20. Hartl DM, Travagli JP. Central compartment neck dissection for thyroid cancer: a surgical technique. World J Surg 2011; 35: 1553-9.

21. Caron NR, Tan YY, Ogilvie JB, Triponez F, Reiff ES, Kebebew E, Duh QY, Clark OH. Selective modified radical neck dissection for papillary thyroid cancer-is level I, II and V dissection always necessary? World J Surg 2006; 30: 833-40.

22. Machens A, Hinze R, Lautenschläger C, Thomusch O, Dralle H. Thy roid carcinoma invading the cervicovisceral axis: routes of invasion and clinical implications. Surgery 2001; 129: 23-8.

23. Sywak M, Cornford L, Roach P, Stalberg P, Sidhu S, Delbridge L. Routine ipsilateral level VI lymphadenectomy reduces postoperative thyroglobulin levels in papillary thyroid cancer. Surgery 2006; 140: 1000-5.

24. Mirghani H, Francois A, Landry G, Hans S, Menard M, Brasnu D. Repeat of lymphatic dissection for thyroid cancers. Ann Otolaryngol Chir Cervicofac 2009; 126: 37-42.

\section{Address for correspondence}

\section{Maciej Pabiszczak, MD}

Department of Otorhinolaryngology, Head and Neck Surgery

Poznan University of Medical Sciences

Przybyszewskiego 49

60-355 Poznań, Poland

e-mail: maciejpabi@poczta.onet.pl

Submitted: 24.03 .2014

Accepted: $\quad 14.04 .2014$ 